\title{
Manipulating the system
}

Descriptions of a biological system are certainly valuable but, in the absence of experimental manipulation, the conclusions that can be drawn about the system are limited. For breast cancer, like many other cancers, characteristic genetic changes have been described. But which genetic changes are causative, and which cellular pathways are affected? These questions, among others, can now be addressed using a manipulable model system for breast cancer, devised in Robert Weinberg's laboratory.

The new work follows on from a landmark paper published by the same laboratory in 1999 - the first successful transformation of human primary cells (embryonic kidney cells and foreskin fibroblasts) by the introduction of oncogenes. The transgenes expressed the SV40 large T and small $t$ antigens, the catalytic subunit of telomerase and an oncogenic version of RAS, HRAS-V12. Elenbaas et al. have now extended this approach to primary human breast epithelial cells and found that the same oncogenes will transform the cells to a tumorigenic phenotype (see pictures) - the cells produce tumours when injected into mice, subcutaneously or into the mammary gland.

Surprisingly, tumour formation was less efficient in the mammary gland than in subcutaneous tissue. This observation hints at the influ- ence of the tissue microenvironment. To investigate this, the authors looked at the effects of mixing transformed epithelial cells with mammary fibroblasts before injection. The result was a marked increase in tumour formation efficiency, which is consistent with the importance of stromal-epithelial interactions and also indicates that this breast cancer system can be used to investigate these interactions.

Karyotypic analysis of the transformed cells revealed that the Myc oncogene was amplified, which is also frequently seen in breast cancer. This is further evidence that the new model system mimics features of naturally occurring breast cancer.

One of the many doors opened by the breast epithelial cell transformation system will allow the investigation of naturally occurring mutations in breast cancer. For example, HER 2 is frequently amplified and overexpressed in breast cancer and is thought to provide a growth-promoting signal. By contrast, RAS is only infrequently mutated, so it will be interesting to see whether HER 2 overexpression can compensate for the RAS transgene used in the model system. No doubt, this work will inspire the development of new experimental systems for other cancers as well.

Mark Patterson

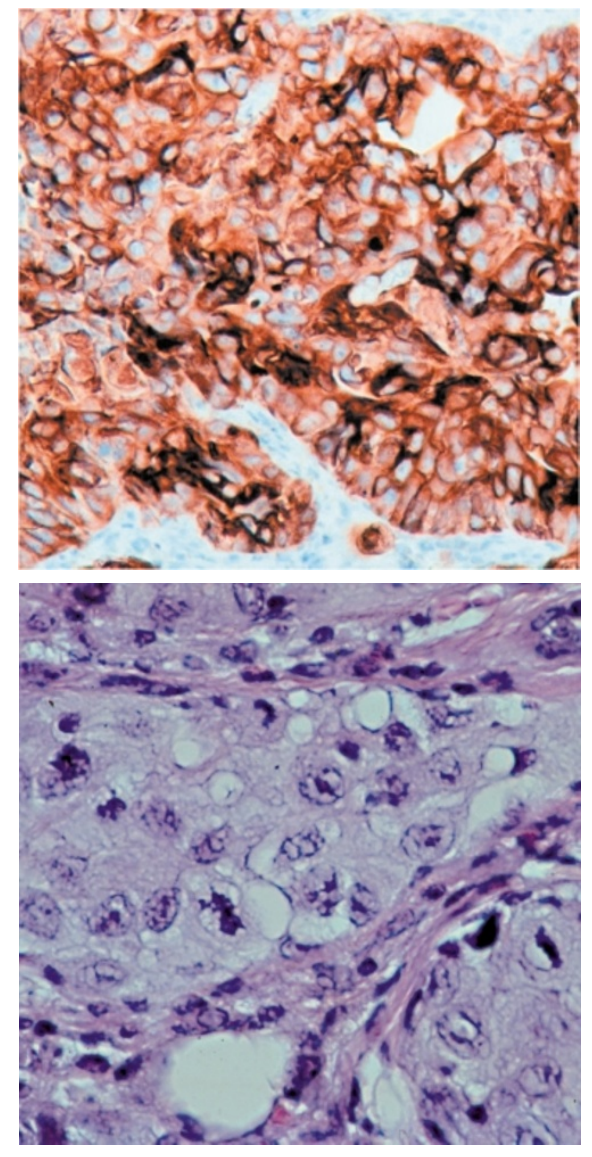

A cytokeratin immunoperoxidase stain (top) shows that the tumour is a carcinoma. The bottom image shows that the epithelial tumour cells and stromal fibroblasts (the elongated pink cells) are closely juxtaposed. Courtesy of Brian Elenbaas (C) CSH Press.

\section{(2) References and links}

ORIGINAL RESEARCH PAPER Elenbaas, B.

et al. Human breast cancer cells generated by oncogenic

transformation of primary mammal epithelial cells. Genes Dev. 15 50-65 (2001)

WEB SITE Robert Weinberg's lab

\section{HUMAN GENETICS}

\section{Alzheimer hotspot on 10}

Unlike early-onset Alzheimer disease — which is caused by mutations in three genes (APP, PSEN1 and PSEN2) - late-onset Alzheimer disease (LOAD) has only one known genetic risk factor, the $\mathrm{E} 4$ allele of apolipoprotein $E$ (APOE4). But only $50 \%$ of LOAD cases carry APOE4 alleles, so the search has been on for additional risk factors. Now, three studies in Science report a major new susceptibility locus for LOAD on chromosome 10.

In 1999, Alison Goate's group identified four possible LOAD linkage regions in a genomewide screen for susceptibility loci in LOADaffected sib pairs (ASPs). In this new study, they typed additional ASPs and chromosome 10 markers and found strongest evidence for linkage close to the marker D10S1211. Their results indicate that this locus might confer as great a risk to $\mathrm{AD}$ relatives as does $A P O E 4$. Ertekin-Tanner et al. also used the 1999 study's results as a starting point to find loci linked to elevated levels of $A \beta 42$ - a cleaved form of $\beta$ amyloid $(\mathrm{A} \beta)$, which is deposited in $\mathrm{AD}$ brains. Plasma $\mathrm{A} \beta 42$ levels are elevated in both LOAD individuals and their cognitively normal relatives, so the authors tested markers from the regions identified in the 1999 study for linkage to plasma $A \beta 42$ levels in relatives of a LOAD individual with high plasma $A \beta 42$. As in the previous study, they found significant linkage to LOAD around D10S1211, indicating that this region might increase disease susceptibility by increasing $A \beta$ levels. However, there are no obvious candidate genes in this region - the closest is the IDE gene, which lies $30 \mathrm{cM}$ away.

Insulin-degrading enzyme (IDE) degrades and clears $A \beta-$ a recent finding that prompted Bertram et al. to test six markers near the presumed location of this gene on chromosome 10q for linkage to $\mathrm{AD}$. In their LOAD sample, three of these markers showed significant linkage to LOAD, one of which lies within $195 \mathrm{~kb}$ of IDE and has an allele that shows significant association with AD. Although this finding does not concur with data from the other two papers - possibly because of different sampling and analytical criteria - together these studies reveal an important new LOAD susceptibility locus. Whether they reflect linkage to one or two chromosome 10 loci remains to be resolved, as does the role of IDE in $\mathrm{AD}$.

\section{Jane Alfred}

\section{(2) References and links}

ORIGINAL RESEARCH PAPERS Bertram, L.

et al. Evidence for genetic linkage of Alzheimer's disease. Science 290, 2302-2303 (2000) | Ertekin-Tanner, N. et al. Linkage of plasma $A \beta 42$ to a quantitative locus on chromosome 10 in late-onset Alzheimer's disease pedigrees. Science $\mathbf{2 9 0}$ 2303-2304 (2000) | Myers, A. et al. Susceptibility locus for Alzheimer's disease on chromosome 10. Science $\mathbf{2 9 0}$ 2304-2305 (2000)

WEB SITE Alzheimer disease education and referral center 